\title{
Intermolecular transfer to polymer in the radical polymerization of n-butyl acrylate
}

Nicholas Ballard, ${ }^{1}$ Shaghayegh Hamzehlou, ${ }^{1}$ José M. Asua ${ }^{1 *}$

${ }^{1}$ POLYMAT and Kimika Aplikatua Saila, University of the Basque Country UPV/EHU, Joxe Mari Korta Zentroa, Tolosa Etorbidea 72, 20018, Donostia/San Sebastián, Spain 


\section{Monte Carlo simulation of seeded semibatch emulsion polymerization of butyl acrylate}

Monte Carlo simulations were conducted using the procedure previously reported. ${ }^{1}$ The kinetic scheme and kinetic rate coefficients of the n-BA polymerization are reported in Scheme S1. Here we briefly explain the important aspects of the simulation, for more details the reader is referred to the mentioned reference.

The KMC algorithm proposed by Gillespie $^{2}$ was implemented. In this algorithm, the experimental rate constants are transformed into stochastical rate constants using a control volume and Avogadro's number. The propensity of each reaction is its rate. The probability of each reaction is defined as its rate over the sum of all reaction rates. Using random numbers and the probabilities, the next reaction and time interval between two reactions are calculated.

In this simulation approach, the initial seed particle volume (a monodisperse particle size distribution is considered) defines the control volume; the solids content defines the control volume of the water phase. The control volume of the water phase and organic phase updates at each time interval according to the feeding rates. Each particle exchanges matter with its own water phase. Monomer partitioning was calculated using partition coefficients which are considered constant trough the reaction. The model accounts for reactions in both aqueous and particle phases. Radical entry was considered to occur by diffusion of surface active oligoradicals and by precipitation of oligoradicals when they become insoluble. In order to get results with statistical meaning, 100 particles were analyzed. 


\begin{tabular}{|c|c|c|}
\hline Reaction & Rate coefficient, $\mathrm{s}^{-1}$ or $\mathrm{L} / \mathrm{mol} / \mathrm{s}$ & Reference \\
\hline$I \stackrel{k_{d}}{\longrightarrow} 2 f R_{0}$ & $\begin{array}{c}k_{d}=8.0 \times 10^{15} \exp (-135.0 / \mathrm{RT}) \\
f=0.6\end{array}$ & 3 \\
\hline$R_{0}+M_{1} \stackrel{k_{i}}{\longrightarrow} R_{1}$ & $k_{p}=2.21 \times 10^{7} \exp (-17.9 / \mathrm{RT})$ & 4 \\
\hline$R_{i}+M_{1} \stackrel{k_{p}}{\longrightarrow} R_{i+1}$ & $k_{p}=2.21 \times 10^{7} \exp (-17.9 / \mathrm{RT})$ & 4 \\
\hline$R_{i}+M_{1} \stackrel{k_{t r m}}{\longrightarrow} P_{i}+R_{1}$ & $k_{t r m}=2.88 \times 10^{5} \exp (-32.6 / \mathrm{RT})$ & 5 \\
\hline$R_{i} \stackrel{k_{b b}}{\longrightarrow} R_{i}^{t, s}$ & $k_{b b}=3.2 \times 10^{10} \exp (-52.3 / \mathrm{RT})$ & 6 \\
\hline$R_{i}+P_{j} \stackrel{k_{t r, p}}{\longrightarrow} P_{i}+R_{j}^{t, l}$ & $k_{t r, p}=0.1-30$ & This work \\
\hline$R_{i}^{t}+M_{1} \stackrel{k_{p}^{t}}{\longrightarrow} R_{i+1}$ & $k_{p}^{t}=1.58 \times 10^{6} \exp (-28.9 / \mathrm{RT})$ & 7 \\
\hline$R_{i}^{t, s} \stackrel{k_{\beta}}{\longrightarrow} R_{i-3}+U_{3}$ & $k_{\beta}=1.47 \times 10^{9} \exp (-55.4 / \mathrm{RT})$ & 6 \\
\hline$R_{i}^{t, s} \stackrel{k_{\beta}}{\longrightarrow} R_{2}+U_{i-2}$ & $k_{\beta}=1.47 \times 10^{9} \exp (-55.4 / \mathrm{RT})$ & 6 \\
\hline$R_{i}^{t, l} \stackrel{k_{\beta}}{\longrightarrow} R_{s}+U_{i-s}$ & $k_{\beta}=1.47 \times 10^{9} \exp (-55.4 / \mathrm{RT})$ & 6 \\
\hline$R_{i}^{t, l} \stackrel{k_{\beta}}{\longrightarrow} R_{i-s}+U_{s}$ & $k_{\beta}=1.47 \times 10^{9} \exp (-55.4 / \mathrm{RT})$ & 6 \\
\hline$R_{i}+U_{j} \stackrel{k_{m a c}}{\longrightarrow} R_{j+i}^{t, l}$ & $k_{m a c}=k p_{11} \times 0.5$ & 8,9 \\
\hline$R_{i}+U_{3} \stackrel{k_{m a c}}{\longrightarrow} R_{j+3}^{t, s}$ & $k_{m a c}=k p_{11} \times 0.5$ & 8,9 \\
\hline$R_{i}+R_{j} \stackrel{c d_{s} * k_{t}}{\longrightarrow} P_{i}+P_{j}$ & $k_{t}=1.3 \times 10^{10} \exp (-8.4 / \mathrm{RT})$ & 8,10 \\
\hline$R_{i}^{t}+R_{j} \stackrel{c d_{s t * k_{t 23}}}{\longrightarrow} P_{i}+P_{j}$ & $k_{t 23}=\sqrt{k_{t 3} \times k_{t}}$ & This work \\
\hline$R_{i}^{t}+R_{j}^{t} \stackrel{c d_{t^{*} k_{t 3}}}{\longrightarrow} P_{i}+P_{j}$ & $k_{t 3}=1.29 \times 10^{7} \exp (-4.0 / \mathrm{RT})$ & 8,11 \\
\hline
\end{tabular}




$$
\begin{array}{rlr}
R_{i}+R_{j} \stackrel{\left(1-c d_{s}\right) * k_{t}}{\longrightarrow} P_{i+j} & k_{t}=1.3 \times 10^{10} \exp (-8.4 / \mathrm{RT}) & \\
R_{i}^{t}+R_{j} \stackrel{\left(1-c d_{s t}\right) * k_{t 23}}{\longrightarrow} P_{i+j} & k_{t 23}=\sqrt{k_{t 3} \times k_{t}} & \text { This work } \\
R_{i}^{t}+R_{j}^{t} \stackrel{\left(1-c d_{t}\right) * k_{t 3}}{\longrightarrow} P_{i+j} & \begin{array}{l}
k_{t 3}=1.29 \times 10^{7} \exp (-4.0 / \mathrm{RT}) \\
c d_{t}=0.9
\end{array} & \\
R_{i_{-} w} \stackrel{k_{a}}{\longrightarrow} R_{i_{-} p} & k_{a}=r_{p} \times k_{a}^{*} \\
k_{a}^{*} & =2.08 \times 10^{4}(1 / \mathrm{s} . d m) & \\
R_{i_{-} p} \stackrel{k_{d}}{\longrightarrow} R_{i_{-} w} & k_{d}=r_{p}^{-2} \times k_{d}^{*} & \\
& k_{d}^{*}=4.5 \times 10^{-13}\left(\mathrm{dm}^{2} / \mathrm{s}\right) & \text { This work }
\end{array}
$$

Scheme S1 The kinetic scheme used in the simulation of seeded semibatch emulsion polymerization of butyl acrylate. I: Initiator molecule. $\mathrm{R}_{0}$ : initiator radical. $\mathrm{R}_{\mathrm{i}}$ : secondary radical with length $i . R_{i}^{t, s}$ : short chain branch tertiary radical with length $i . R_{i}^{t, l}$ : long chain branch tertiary radical with length $i$. $P_{i}$ : dead polymer with length $i$. $U_{i}$ : macromonomer with length $i . R_{i_{-} w}$ and $R_{i_{-} p}$ are radical species in the water and polymer phases respectively. M1 represents BA (activation energies are in $\mathrm{kJ} / \mathrm{mol}$ )

\section{References:}

(1) Hamzehlou, S.; Reyes, Y.; Leiza, J. R. Modeling the mini-emulsion copolymerization of N-butyl acrylate with a water-soluble monomer: A Monte Carlo approach Ind. Eng. Chem. Res. 2014, 53, 8996-9003.

(2) Gillespie, D. T. Stochastic simulation of chemical kinetics. Annu. Rev. Phys. Chem. 2007, 58, 35-55. 
(3) Lansalot, M.; Davis, T. P.; Heuts, J. P. A. RAFT Miniemulsion Polymerization: Influence of the Structure of the RAFT Agent Macromolecules 2002, 35, 75827591.

(4) Asua, J. M.; Beuermann, S.; Buback, M.; Castignolles, P.; Charleux, B.; Gilbert, R. G.; Hutchinson, R. A.; Leiza, J. R.; Nikitin, A. N.; Vairon, J. P.; van Herk, A. M. Critically Evaluated Rate Coefficients for Free-Radical Polymerization, 5

Propagation Rate Coefficient for Butyl Acrylate Macromol. Chem. Phys. 2004, 205, 2151-2160.

(5) Maeder, S.; Gilbert, R. G. Measurment of Transfer Constant for Butyl Acrylate FreeRadical Polymerization Macromolecules 1998, 31, 4410-4418.

(6) Hamzehlou, S.; Ballard, N.; Reyes, Y.; Aguirre, A.; Asua, J. M.; Leiza, J. R. Analyzing the discrepancies in the activation energies of the backbiting and $\beta$ scission reactions in the radical polymerization of n-butyl acrylate Polym. Chem. 2016, 7, 2069-2077.

(7) Nikitin, A. N.; Hutchinson, R. A.; Buback, M.; Hesse, P. Determination of Intramolecular Chain Transfer and Midchain Radical Propagation Rate Coefficients for Butyl Acrylate by Pulsed Laser Polymerization Macromolecules 2007, 40, 86318641 .

(8) Nikitin, A. N.; Hutchinson, R. A.; Wang, W.; Kalfas, G. A.; Richards, J. R.; Bruni, C. Effect of Intramolecular Transfer to Polymer on Stationary Free-Radical Polymerization of Alkyl Acrylates, 5 - Consideration of Solution Polymerization up to High Temperatures Macromol. React. Eng. 2010, 4, 691-706.

(9) Wang, W.; Nikitin, A. N.; Hutchinson, R. A. Consideration of Macromonomer Reactions in $\mathrm{n}$-Butyl Acrylate Free Radical Polymerization Macromol. Rapid Commun. 2009, 30, 2022-2027.

(10) Barth, J.; Buback, M.; Hesse, P.; Sergeeva, T. Termination and Transfer Kinetics of Butyl Acrylate Radical Polymerization Studied via SP-PLP-EPR Macromolecules 2010, 43, 4023-4031.

(11) Arzamendi, G.; Leiza, J. R. Molecular Weight Distribution (Soluble and Insoluble Fraction) in Emulsion Polymerization of Acrylate Monomers by Monte Carlo Simulations Ind. Eng. Chem. Res. 2008, 47, 5934-5947. 